Edith Cowan University

Research Online

Research outputs 2012

$1-1-2012$

\title{
Strength Gains: Block Versus Daily Undulating Periodization Weight Training Among Track and Field Athletes
}

\author{
Keith Painter \\ Guy Haff \\ Edith Cowan University \\ Michael Ramsey \\ Jeff McBride \\ Travise Triplett
}

See next page for additional authors

Follow this and additional works at: https://ro.ecu.edu.au/ecuworks2012

Part of the Sports Sciences Commons

10.1123/ijspp.7.2.161

This is an Author's Accepted Manuscript of: Painter, K., Haff, G. G., Ramsey, M., McBride, J., Triplett, T., Sands, W., Lamont, H., Stone, M., \& Stone, M. (2012). Strength Gains: Block Versus Daily Undulating Periodization Weight Training Among Track and Field Athletes. International Journal of Sports Physiology and Performance, 7(2), 161-169. Available here (c) Human Kinetics, Inc.

This Journal Article is posted at Research Online.

https://ro.ecu.edu.au/ecuworks2012/497 


\section{Authors}

Keith Painter, Guy Haff, Michael Ramsey, Jeff McBride, Travise Triplett, William Sands, Hugh Lamont, Margaret Stone, and Michael Stone 


\title{
Strength Gains: Block Versus Daily Undulating Periodization Weight Training Among Track and Field Athletes
}

\author{
Keith B. Painter, Gregory G. Haff, Mike W. Ramsey, Jeff McBride, Travis Triplett, \\ William A. Sands, Hugh S. Lamont, Margaret E. Stone, and Michael H. Stone
}

\begin{abstract}
Recently, the comparison of "periodized" strength training methods has been a focus of both exercise and sport science. Daily undulating periodization (DUP), using daily alterations in repetitions, has been developed and touted as a superior method of training, while block forms of programming for periodization have been questioned. Therefore, the purpose of this study is to compare block to DUP in Division I track and field athletes. Thirty-one athletes were assigned to either a 10-wk block or DUP training group in which sex, year, and event were matched. Over the course of the study, there were 4 testing sessions, which were used to evaluate a variety of strength characteristics. Although performance trends favored the block group for strength and rate of force development, no statistically significant differences were found between the 2 training groups. However, statistically different $(P \leq .05)$ values were found for estimated volume of work (volume load) and the amount of improvement per volume load between block and DUP groups. Based on calculated training efficiency scores, these data indicate that a block training model is more efficient than a DUP model in producing strength gains.
\end{abstract}

Keywords: volume load, intensity, rate of force development, training efficiency

Programming methods (models) of strength/power training have been developed, with each model purporting to represent a form of periodization and purporting to make advances over block forms of periodization. Periodization in block form has been suggested to be linear in nature ${ }^{1}$ and thus results in too little variation for optimum performance adaptation. Conversely, daily undulating periodization (DUP) models use a form of variation in which repetitions are altered each training session throughout the training week, creating greater variation in training stimulus, which has been suggested to produce superior physiological and performance adaptations. ${ }^{2}$ However, few comparison studies have examined differences among these models. Critical analyses of these studies ${ }^{3-9}$ reveal shortcomings that potentially reduce the ability to clearly differentiate between models

Painter, Ramsey, Lamont, ME Stone, and MH Stone are with KLSS/Center of Excellence for Sport Science and Coach Education, East Tennessee State University, Johnson City, TN. Haff is with the School of Exercise, Biomedical and Health Sciences, Edith Cowan University, Joondalup, WA, Australia. McBride and Triplett are with the Dept of Health, Leisure and Exercise Science, Appalachian State University, Boone, NC. Sands is with the Kinesiology Dept, Mesa State University, Grand Junction, CO. and thus determine their applicability to well-trained and athlete populations. For example, the use of untrained or recreationally trained subjects makes it more difficult to detect adaptations to short-term training associated with trained athletes; only one study used relatively advanced athletes. $^{5}$

The block model depends upon several levels of variation, including the use of heavy and light days of training in which the amount of work performed (volume load) is reduced for the light day. This type of loading paradigm has the potential to enhance recovery and adaptation processes, leading to a superior performance. ${ }^{10,11}$ Only one comparison study reported an attempt to use heavy and light days in a block model. ${ }^{5}$ In addition, most studies examining DUP models also used training requiring subjects to perform most or all exercise with repetition maximum (RM) ranges that necessitate relatively consistent training to failure. Training to failure produces a constant relative maximum intensity, potentially negating any differences between groups based on heavy- and light-intensity days. ${ }^{11}$ Furthermore, even though intensity (load) was decreased on some days, the repetitions (and volume load) were increased, which resulted in an overall increased workload.

Another issue confounding the ability to interpret DUP models is that some researchers attempted to equalize training volume and intensity, usually by equalizing 
repetitions, a method that tends to obviate the necessary alterations in training variables (ie, volume and intensity) that are typically employed in block methods. Only one of the studies attempted to track alterations in work estimated by volume load. ${ }^{6}$ None of the studies estimated program efficiency. Efficiency can be calculated as follows: Performance gain divided by work invested. By estimating training efficiency, it may be possible to detect the effectiveness of a training program by exploring how an estimate of work invested (volume load) relates to strength gains.

Few studies compare block and DUP programming models and the results are unclear as to the efficacy of these programming models. Thus, the purpose of this study was to compare a DUP versus a block model of strength/power training using NCAA Division I track and field athletes during a 10-week fall-semester preparation-phase program. Comparisons were made between programming models for maximum isometric strength, instantaneous forces, rate of force development, 1-repetition maximum (1 RM) parallel squat, and differences in the volume load. In addition, in order to further evaluate the effectiveness of each programming model, training efficiency was calculated.

\section{Methods}

\section{Athletes}

Thirty-two college track athletes, 18-22 years of age, initially participated in this study ( 23 men, 9 women). Before the initiation of the study, sport medicine staff carried out athlete health screenings that included a medical physical examination and questionnaires concerning any current injury. Athletes were orally informed of the requirements and risks of the study and then read and signed institutional review board-approved informedconsent documents before study initiation. Athletes were divided equally between groups based on event, sex, and year (freshmen or returners). No athletes performing primarily endurance activities were used in the study. Before study participation, all athletes were deemed healthy and approved for practice by the sport medicine staff. During the study, if an injury occurred, the sport medicine staff examined the athlete and documented the type and extent of the injury before continued participation. Exclusion criteria were missing three training sessions for any reason, missing any testing session, noncompliance with the prescribed protocol, and performing physical training outside of normal practice or strength training sessions. At the conclusion of the study, 6 athletes ( 1 male, 1 female from the block group; 3 males, 1 female from the DUP group) had been excluded. Initial demographic data for the athletes completing the study were block $(\mathrm{n}=14)$, height $=176.9 \pm 11.3 \mathrm{~cm}$, age $=19.9 \pm 1.2$ years, body mass $=86.1 \pm 30.9 \mathrm{~kg}, \%$ fat $=18.0 \pm 15.5$, and DUP $(\mathrm{n}$ $=12)=177.2 \pm 5.6$, age $=19.4 \pm 0.8$ years, body mass $=80.7 \pm 18.1, \%$ fat $=14.5 \pm 8.5$. There were no initial statistical differences between groups.

\section{Experimental Approach to the Problem}

This study was part of an ongoing athlete research and monitoring program, in which sport coaches had control of sport practices; lifestyle, such as eating habits, was not controlled. The study was a counterbalanced design in which a group of Division I (D-I) college track and field athletes were divided into two resistance training groups, performing either a block model or a DUP program for a 10 -week training period. Additional training (eg, sprint or event practice) was identical for each group. Measurements were made at 0 weeks (T1), after 4 weeks (T2), 8 weeks (T3), and 11 weeks (T4).

Training programs were developed based on extensive literature review and were reviewed by multiple strength coaches in order to ensure that they were being applied appropriately. Repetition maximum zones were used only with DUP in specific exercises based on previous research ${ }^{3-9}$ and the text by Kraemer and Fleck. ${ }^{2}$ By equating volume and/or intensities, it is likely fundamental differences associated with each training model are obviated..$^{3,4,6-9}$ Training volumes were not equated for several reasons. First, it is likely that equating the volumes between the two interventions creates a training paradigm and training scenario that do not accurately represent actual athlete training practices. Second, by equating workload between the training groups, one or both of the interventions are potentially using a training scheme in which specific variables, such as the training load, number of sets, and repetitions, are not optimally applied. Problems with equating training volume have been largely overlooked in the scientific literature. ${ }^{7-9,12}$ While experimental controls have always played a dominant role in the conduct of investigations, determining which variables require control and which are simply measured and/or randomized is vitally important for application to external validity. Too often, experimental controls are imposed that make the experiment so different from the reality of training that one can only conclude that the results apply only to the experimental condition. As groups were matched by number of athletes, by event, sex, and non-strength training variables, the only major differences were those represented by the strength training programs.

\section{Methodology}

At each session, coaches recorded the loads lifted and repetitions completed for each set. Volume load and intensity were calculated using custom Excel spreadsheets. ${ }^{13}$ Athletes were surveyed throughout the study concerning additional exercise performed outside of normal training. Practice data were monitored for accuracy and verified with the sport coaching staff. These data were used to ensure that both groups were performing approximately equal amounts of non-strength training work. Measurements were made at 0 weeks (T1) and after 4 weeks (T2), 8 weeks (T3), and 11 weeks (T4). 


\section{Calculation of Volume Load and Intensity}

Volume load (VL) is an easily calculated estimate of work performed ${ }^{11,13}$ and was calculated with the following equation:

$$
\text { Volume load }(\mathrm{kg})=\text { sets } \times \text { repetitions } \times \text { load }
$$

Strength training intensity (TI) was estimated by calculating the average load lifted, represented by the following equation: ${ }^{13}$

$$
\begin{gathered}
\text { Training intensity }(\mathrm{kg})= \\
\text { volume load }(\mathrm{kg}) / \text { total repetitions }
\end{gathered}
$$

Once the VL is calculated, training efficiency (TE) can be determined by dividing the gain score by the amount of work performed:

Training efficiency = gain score/volume load

Taken collectively, the VL, TI, and TE can be used to better evaluate training interventions and give insight into the outcomes achieved by the training plans.

\section{Testing}

Testing occurred at the beginning of the week (Monday and Tuesday), on weeks 1 (T1), 4 (T2), 8 (T3), and 11 (T4). Testing dates corresponded to the start of a new block of training for the block group. Athletes were familiarized with the testing and training protocols on multiple occasions before T1. ${ }^{14}$

Testing consisted of hydration status, body composition, 1-RM parallel squat, and isometric midthigh pulls (MTPs). A testing session replaced the training session for that day and no other activity was permitted. Testing began on Monday of each testing week (the 1-RM squats took place on Tuesday).

\section{Hydration}

Hydration was estimated from urinary specific gravity (USG) using refractometry. Hydration status has implications for fatigue, performance, and cognitive abilities and could affect test results. Hydration was also measured on a random basis throughout the study to ensure the athletes were maintaining a hydrated status.

\section{Physical Characteristics}

Height was measured using a stadiometer. Body mass was measured using an electronic scale. Body composition was assessed using plethysmography (BodPOD, Concord, CA).

\section{Strength Measures}

Isometric Strength. After a standard warm-up protocol, athletes performed an isometric MTP. ${ }^{14}$ The MTP was performed on a custom isometric rack that allowed for incremental adjustments (Sorinex, Irmo SC). MTPs were performed on a force plate (Rice Lake Weighing
Systems, Rice Lake, WI) sampling at $1000 \mathrm{~Hz}$. This testing procedure is relatively rapid ( $10 \mathrm{~min} /$ athlete), has a high reliability $\left(n=200 ; \operatorname{ICC}_{\mathrm{IPF}} \geq .99\right.$ and ICC $\alpha_{\mathrm{RFD}} \geq$ .91 ), and shows strong relationships to typical dynamic measures of 1-RM. ${ }^{15,16}$

Data were recorded and analyzed using LabView 8 software (National Instruments, Upper Saddle River, NJ). Data were smoothed using a moving average and filtered using a low-pass Butterworth filter with a cutoff frequency of $100 \mathrm{~Hz}$. The force-time measures were analyzed for peak force (IPF), rate of force development (RFD) from 0 to 200 milliseconds, and instantaneous force at 50 (F50), 90 (F90), and 250 milliseconds (F250). ${ }^{14}$

1-RM Parallel Squat. The 1-RM parallel squat (1RMSQ) was used as a training-specific measure of dynamic strength. Testing took place on Tuesday morning, thus allowing recovery from the previous day (iso-pull). Testing was carried out with Olympic standard barbells and weights. Athletes warmed up in a progressive manner. Typically, 5 to 8 sets of squats were needed to reach 1-RM values. Each athlete was allowed to use his or her normal squatting stance/positions, which had been previously established. Test-retest reliability for the 1 RMSQ has been previously established in our laboratory $(\mathrm{n}=200, \mathrm{ICC} \alpha=.92)$.

\section{Training Programs}

Athletes resistance trained $3 \mathrm{~d} / \mathrm{wk}$ for 10 weeks; each session was completed within 1 hour. The basic structure of the block program and exercises employed are presented in Table 1.

The training plan incorporated heavier and lighter days into the weekly training structure; for example, Monday was always heavier than Friday (Table 1). This was accomplished by decreasing the loads (intensity) on Friday by approximately $15 \%$. This alteration in loading was carried out to aid in managing fatigue and to provide contraction velocity variation.

When developing the DUP program (Table 2), much of the literature is unspecific in defining and describing the construction and application of the programming model. After consulting multiple sources, ${ }^{1-9,11}$ it was ascertained that each day in DUP would more or less be congruent to a phase of the block program. Thus, each of the 3 days of lifting in the DUP group (Table 2) corresponded to the 3 distinct blocks setup in the block program, each day having a similar focus (ie, strengthendurance, strength, power). The next challenge in the development of the DUP program was condensing each block into 1 training day. Intensity variations for the DUP program utilized the RM zone method, which is typically recommended for DUP training plans, and was used in comparison studies. ${ }^{1-9}$ Repetition maximum zone training was also applied to the selection of exercises for each day of the DUP training program. However, not all DUP exercises were performed to RM (failure values). Power-oriented exercises such as pulling movements and loaded jumps were performed with a load requiring 
Table 1 The Traditional Group Training Plan

\begin{tabular}{|c|c|c|c|c|c|c|}
\hline \multirow[b]{2}{*}{ Block and emphasis } & \multirow[b]{2}{*}{ Week } & \multirow[b]{2}{*}{ Sets } & \multirow[b]{2}{*}{ Repetitions } & \multicolumn{3}{|c|}{ Intensity } \\
\hline & & & & Monday & Wednesday & Friday \\
\hline \multirow{3}{*}{ 1: Strength/Endurance } & 1 & 3 & 10 & M & ML & $\mathrm{L}$ \\
\hline & 2 & 3 & 10 & MH & M & $\mathrm{L}$ \\
\hline & 3 & 3 & 10 & $\mathrm{H}$ & M & ML \\
\hline \multirow{3}{*}{ 2: Strength } & 4 & 3 & $5^{*}$ & M & M & $\mathrm{L}$ \\
\hline & 5 & 3 & $5^{*}$ & MH & MH & $\mathrm{L}$ \\
\hline & 6 & 3 & $3 *$ & $\mathrm{H}$ & $\mathrm{H}$ & ML \\
\hline \multirow{4}{*}{ 3: Power } & 7 & 3 & $2^{*}$ & $\mathrm{VH}$ & $\mathrm{H}$ & ML \\
\hline & 8 & 5 & 5 & M & $\mathrm{H}$ & $\mathrm{L}$ \\
\hline & 9 & 3 & $3 *$ & $\mathrm{H}$ & MH & ML \\
\hline & 10 & 1 & $3 *$ & VH & M & ML \\
\hline & \multicolumn{6}{|c|}{ Exercises } \\
\hline Day & Block 1 & & Block 2 & Block 3 & & \\
\hline \multirow[t]{4}{*}{ Monday/Friday } & Back squat & & Back squat* & $1 / 4$ back sc & & \\
\hline & Behind-neck press & & Push press* & Weighted j & & \\
\hline & Bench press & & Incline press* & Push jerk* & & \\
\hline & & & & Incline dun & 11 press & \\
\hline \multirow[t]{5}{*}{ Wednesday } & Power snatch & & Power snatch & Power snat & & \\
\hline & Clean grip shrug & & Clean grip shrug & Midthigh $\mathrm{p}$ & & \\
\hline & Midthigh pull & & Mid high pull* & Stiff-leg de & & \\
\hline & Stiff-leg deadlight & & Stiff-leg dead lift & & & \\
\hline & Dumbbell rows & & Dumbbell rows & & & \\
\hline
\end{tabular}

Note: *Down set of $1 \times 5$ at approximately $60 \%$ of target sets; $\$ 0-30 \%$ of body mass. Intensities were based on a projected maximum for sets and repetitions (based on Stone et al, 2007). $\mathrm{L}=$ light (approximately $65-70 \%$ of 1-RM); ML = moderate light (approximately $70-75 \%$ of $1-\mathrm{RM}$ ), $\mathrm{M}=$ moderately (approximately $75-80 \%$ of $1-\mathrm{RM}), \mathrm{MH}=$ moderately heavy.

Table 2 The Daily Undulating Group Training Plan

\begin{tabular}{lcccc}
\hline Day & Emphasis & Sets & Repetitions & Intensity \\
\hline Monday & Strength/Endurance & 3 & $8-12$ & $8-12 \mathrm{RM}$ \\
Wednesday & Strength & 3 & $5-7$ & $5-7 \mathrm{RM}$ \\
Friday & Power & 3 & $3-5$ & $3-5 \mathrm{RM}$ \\
\multicolumn{4}{c}{ Exercises } \\
\hline Monday & Wednesday & Friday \\
\hline Back squat & Back squat & 1/4 back squat \\
Midthigh pull & Clean grip shrug & Midthigh pull \\
Behind-neck press & Push press & Weighted jump§ \\
Bench press & Incline bench press & Push jerk \\
Dumbbell row & Dumbbell row & Stiff-leg dead lift \\
\hline
\end{tabular}

Note: $\mathrm{RM}=$ repetition maximum; $\S 0-30 \%$ of body mass . 
a reasonable effort (as determined by the coach) using the middle of the RM repetition range, but not to failure.

Importantly, volume and intensity were not purposefully equated. Based upon theories of periodization, the actual differences noted in these variables are key factors that are central to the differences between the 2 training program models. As such, in the present study, the block method manipulates VL and TI within and across weeks; however, the DUP protocol manipulates repetitions within weeks.

Every training session was monitored by knowledgeable coaches to ensure exercise technique was appropriate and training program compliance was maintained. In order to maximize the effectiveness of the coaching and monitoring of the training sessions, each coach was assigned 3 or 4 athletes. To remove training bias, coaches were alternated randomly between training groups on a biweekly basis.

Trained athletes often respond differently than untrained, semitrained, or well-trained individuals. Part of the reason for this is that athletes are not typically performing resistance training in isolation and perform additional forms of training outside of the weight room. The present study addresses this problem by using college track and field athletes during fall preparation training. Outside practice and conditioning were constant factors for these athletes, thus the strength-training methods represented major volume and intensity alterations among the athletes.

\section{Statistical Analyses}

Data were analyzed with SPSS (version 16.0; SPSS, Inc. Chicago, IL.). Multiple $2 \times 4$ repeated-measures ANOVAs were used to determine statistical differences between groups and the measurement times for all tested variables. A $2 \times 10$ ANOVA was used for analyzing the volume load for each of the 10 weeks of training. Follow-up one-way ANOVAs were performed to determine where significant differences existed. Allometric scaling of force (strength) was used to normalize affects of body size.

$$
\begin{gathered}
\text { Allometric scaling }= \\
\text { isometric force/(body mass } \left.(\mathrm{kg})^{2 / 3}\right)
\end{gathered}
$$

Correlations were calculated using Pearson's $r$ (statistical significance was $P \leq .05, r=.34$ ). Effect sizes were calculated. No statistical differences were noted between sexes for gain in performance. Thus, data is presented as groups (DUP vs block).

\section{Results}

Body mass of the combined groups increased over time (T1 to T4) $P \leq .05$. Block body mass increased from $86.1 \pm 30.9$ to $87.0 \pm 30.4 \mathrm{~kg}$ and DUP increased from $80.7 \pm 18.1$ to $83.7 \pm 19.1$. However, over the 10 weeks, there were no statistical differences between groups, except for block, which was more efficient, and used fewer total repetitions $(P=.001: \sim 52 \%)$ and VL $(P=$ $.0004 ; \sim 35 \%)$. Training intensity showed no statistical differences by week or as an average over $10 \mathrm{wk}$ (block $=60 \pm 14 \mathrm{~kg}$ vs DUP $=66 \pm 12 \mathrm{~kg}$ ); thus, only the VL was statistically altered.

\section{Maximum Strength}

Isometric peak force (IPF), allometrically scaled isometric peak force IPFa, 1RMSQ, and allometrically scaled

\begin{tabular}{|c|c|c|c|c|}
\hline \multirow[b]{2}{*}{ Group and testing time } & \multicolumn{2}{|c|}{ Isometric Peak Force (kg) } & \multicolumn{2}{|c|}{ Back Squat 1-RM (kg) } \\
\hline & & Allometrically scaled & & Allometrically scaled \\
\hline \multicolumn{5}{|l|}{ Traditional } \\
\hline time 1 & $4610.0 \pm 1636.0$ & $237.0 \pm 50.0$ & $129.0 \pm 41.0$ & $1.54 \pm 1.36$ \\
\hline time 2 & $4699.0 \pm 1651.0$ & $239.0 \pm 38.0$ & $133.0 \pm 42.0$ & $1.58 \pm 1.38$ \\
\hline time 3 & $4904.0 \pm 1544.0$ & $249.0 \pm 38.0$ & $138.0 \pm 41.0$ & $1.68 \pm 1.38$ \\
\hline time 4 & $5304.0 \pm 1709.0$ & $271.0 \pm 47.0$ & $134.0 \pm 41.0$ & $1.61 \pm 1.41$ \\
\hline \multicolumn{5}{|l|}{ Daily undulating } \\
\hline time 1 & $4032.0 \pm 1084.0$ & $215.0 \pm 41.0$ & $131.0 \pm 36.0$ & $1.62 \pm 1.91$ \\
\hline time 2 & $4277.0 \pm 1057.0$ & $225.0 \pm 37.0$ & $139.0 \pm 38.0$ & $1.69 \pm 2.10$ \\
\hline time 3 & $4595.0 \pm 840.0$ & $241.0 \pm 34.0$ & $143.0 \pm 38.0$ & $1.72 \pm 2.10$ \\
\hline time 4 & $4625.0 \pm 1144.0$ & $242.0 \pm 43.0$ & $133.0 \pm 40.0$ & $1.62 \pm 2.10$ \\
\hline
\end{tabular}

Table 3 Maximal Strength Data (Mean \pm SD) for the Mid-Thigh Pull and Back Squat Tests

Note: $\mathrm{RM}=$ repetition maximum. 


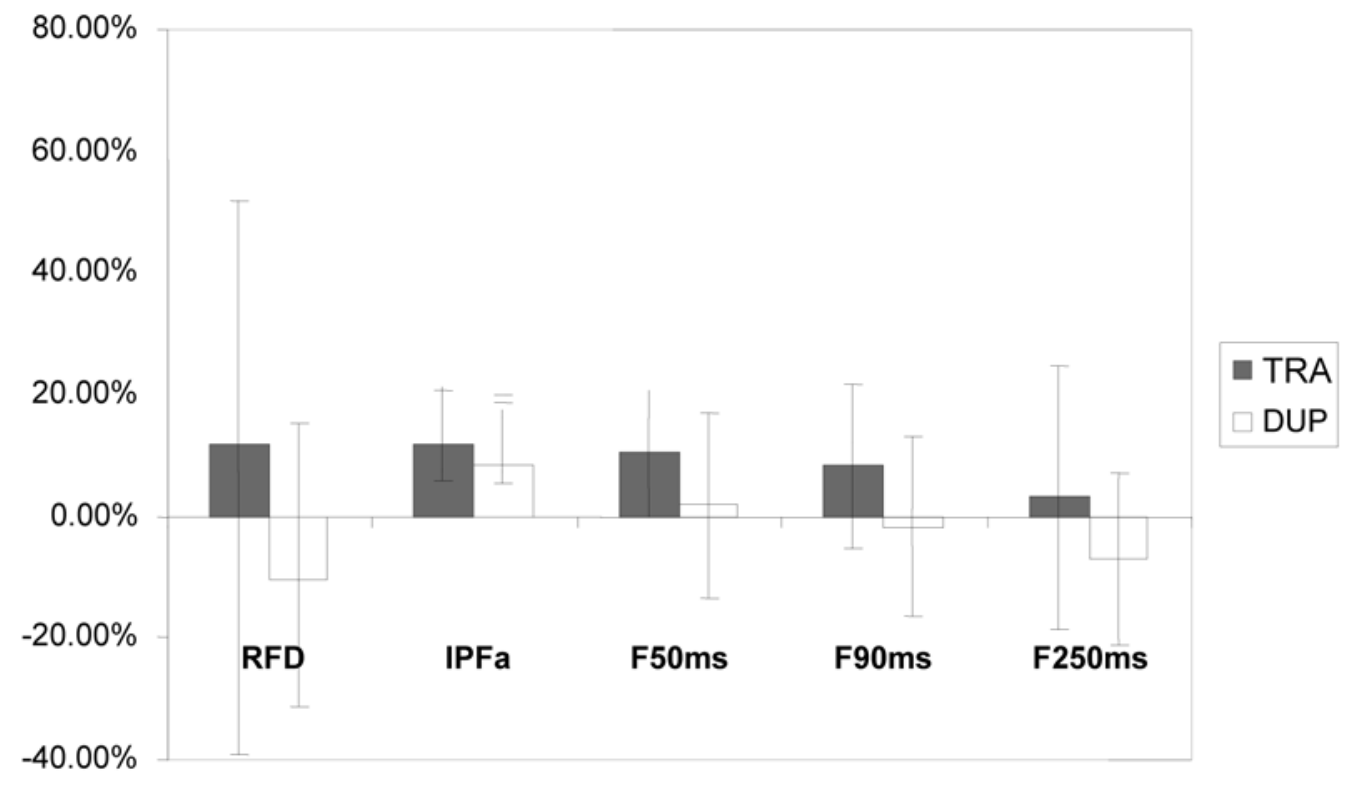

Figure 1 - Net percent differences in isometric RFD and forces (T1 vs T4). TRA = traditional group; DUP, daily undulating group.

squat (SQa) are presented in Table 3, for each group at each test session. Values for 1RMSQ instantaneous and maximum forces improved over time $(P \leq .05)$.

Net changes for RFD and force are shown in Figure 1. Although there were no statistical differences for force between groups, the block group showed a slight improvement for all force measurements. For T1-T4, the block effect size was 0.73 (gain $=14.7 \%$ ) for IPFa and 0.41 for IPF (gain $=15.1 \%$ ). The DUP effect size was 0.63 (gain $=12.1 \%)$ for $\mathrm{IPFa}$ and $0.52($ gain $=$ $14.7 \%$ ) for IPF. The effect size for the block RFD was trivial (0.001) and the effect size for DUP was -0.20 . From $\mathrm{T} 1$ to $\mathrm{T} 4$, the block model showed small positive percent gains $($ absolute $=3.9 \%$; scaled squat $=4.5 \%$ ), but this was not the case for the DUP (1.5\% absolute; $0 \%$ scaled) The squat effect sizes, although larger for block, were trivial to small for both groups from $\mathrm{T} 1$ to T4. (Table 3).

Strong correlations for the combined groups were noted between the IPF and the 1-RM squat T1 $(r=.67)$, $\mathrm{T} 2(r=.68), \mathrm{T} 3(r=.73)$, and T4 $(r=.68)$.

\section{Repetitions and Volume Load}

Data revealed a markedly $(P \leq 0.05)$ higher number of repetitions (52\%) for the DUP group (Table 4) and VL was statistically different in each of the last $7 \mathrm{wk}$ of training in the DUP group (Table 5). The total work (estimated by VL) was approximately 35\% higher for the DUP compared with the block.

As a result of the differences in volume of work (VL), the efficiency in gain scores are markedly different. Across time, the block model was progressively more efficient than the DUP $(P \leq .05)$ (Figure 2). Similar findings were noted for the 1RM squat.

\section{Discussion}

The primary finding was that the block training method was more efficient for improving maximal strength and the rate of force development. Specifically, the block group demonstrated significantly greater increases in the $1 \mathrm{RSQ}$, and isometric values when differences in volume load were considered.

The theoretical basis for DUP is that more training variety should stimulate greater gains in strength and related characteristics. For example, Kraemer et al ${ }^{2}$ compared DUP to a linear training program (2-3 sets of $8-10$ RM each day) and found that greater variation produced greater strength gains. Previous investigations ${ }^{1-9}$ of block forms of periodized strength training versus DUP have resulted in equivocal statistical results. These studies are not without problems, for example, equating volume and intensity, ${ }^{8,9}$ unclear descriptions of training groups, ${ }^{7}$ and unreported effect sizes. ${ }^{3,5}$ Indeed, when effect sizes and percentage gains are considered, many of these studies agree with the findings of Hartmann et $\mathrm{al},{ }^{4}$ suggesting that the block method is more efficacious.

Using freshmen American football players, Hoffman et $\mathrm{al}^{5}$ compared what they termed linear periodization (LP) to a DUP model and found no statistical differences in most variables. However, medicine ball throws increased in the LP group $(P \leq .05)$, indicating improved explosiveness. Calculations (not presented by Hoffman et $\left.\mathrm{al}^{5}\right)$ of the effect size (ES $=1.43$ vs 0.74$)$ and the percent gain $(20.9 \%$ vs $11.1 \%)$ suggest that the LP group actually produced superior gains in the squat compared with the DUP group.

Note that the block method dropped squats from training during the last $3 \mathrm{wk}$. Dropping squats and substituting $1 / 3$ squats and weighted jumps during the 
Table 4 Comparison of Repetitions (Mean \pm SD) Accomplished by the Traditional and Daily Undulating Groups

\begin{tabular}{lcccc}
\hline Week & Traditional group & Daily undulating group & $\boldsymbol{P}$ & ES \\
\hline 1 & $264.3 \pm 79.5$ & $240.8 \pm 40.7$ & .360 & 0.03 \\
2 & $404.5 \pm 46.0$ & $476.8 \pm 63.4$ & .002 & 0.32 \\
3 & $418.7 \pm 35.7$ & $440.3 \pm 37.4$ & .138 & 0.09 \\
4 & $174.7 \pm 31.6$ & $227.0 \pm 27.9$ & $\mathbf{. 0 0 1}$ & 0.45 \\
5 & $214.7 \pm 62.8$ & $315.7 \pm 102.4$ & $\mathbf{. 0 0 4}$ & 0.29 \\
6 & $196.9 \pm 35.6$ & $450.3 \pm 97.3$ & $\mathbf{. 0 0 1}$ & 0.78 \\
7 & $173.3 \pm 42.7$ & $390.4 \pm 102.0$ & $<.001$ & 0.69 \\
8 & $215.1 \pm 30.6$ & $238.5 \pm 48.2$ & .137 & 0.09 \\
9 & $169.9 \pm 28.9$ & $447.5 \pm 102.4$ & $<.001$ & 0.80 \\
10 & $255.5 \pm 35.2$ & $460.6 \pm 70.7$ & $<.001$ & 0.80 \\
Total & $2487.5 \pm 234.8$ & $3687.9 \pm 377.4$ & $<.001$ & 0.80 \\
\hline
\end{tabular}

Note: statistically significant values are in bold.

Table 5 Comparison of Volume Load (Mean \pm SD) Accomplished by the Traditional and Daily Undulating Group

\begin{tabular}{lcccc}
\hline Week & Traditional group & Daily undulating group & $\boldsymbol{P}$ & ES \\
\hline 1 & $14,072.8 \pm 6054.3$ & $14,703.9 \pm 4002.6$ & .76 & 0.004 \\
2 & $21,584.4 \pm 7826.4$ & $26,410.7 \pm 5830.5$ & .09 & 0.112 \\
3 & $21,172.8 \pm 6169.0$ & $26,127.4 \pm 7021.3$ & .06 & 0.132 \\
4 & $11,710.5 \pm 5354.0$ & $16,319.3 \pm 4349.6$ & $\mathbf{. 0 2}$ & 0.189 \\
5 & $13,569.9 \pm 5956.8$ & $20,234.5 \pm 7438.5$ & $\mathbf{. 0 2}$ & 0.211 \\
6 & $12,596.1 \pm 3993.6$ & $29,527.1 \pm 10,401.1$ & $\mathbf{. 0 0 1}$ & 0.575 \\
7 & $10,893.6 \pm 3716.3$ & $25,733.1 \pm 9689.6$ & $\mathbf{. 0 0 1}$ & 0.545 \\
8 & $13,308.6 \pm 3524.1$ & $20,499.5 \pm 7,161.3$ & $\mathbf{. 0 0 2}$ & 0.318 \\
9 & $11,246.8 \pm 3564.7$ & $31,325.1 \pm 12,378.2$ & $\mathbf{. 0 0 1}$ & 0.590 \\
10 & $15,575.6 \pm 6095.9$ & $30,326.9 \pm 10,042.1$ & $\mathbf{. 0 0 1}$ & 0.471 \\
Total & $145,731.0 \pm 44,612.5$ & $241,207.5 \pm 67,657.2$ & $\mathbf{. 0 0 0 4}$ & 0.437 \\
\hline
\end{tabular}

Note: statistically significant values are in bold.

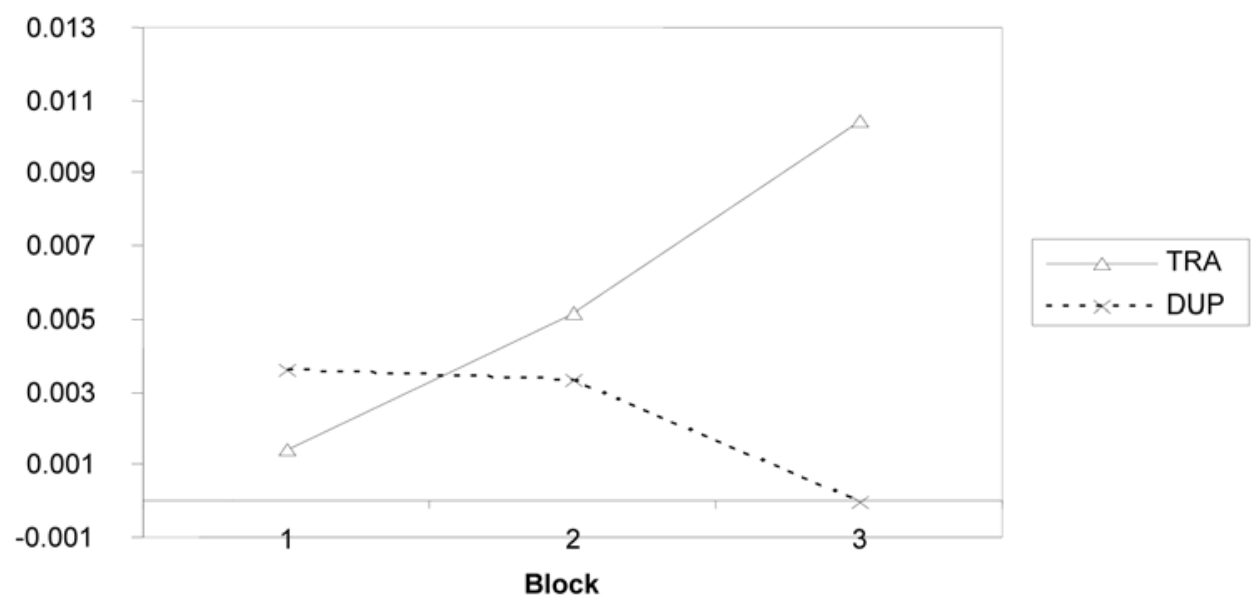

Figure 2 - Gain is IPF/volume load $(\mathrm{kg})$. TRA = traditional group; DUP, daily undulating group. 
final phase is believed to reduce fatigue and emphasize power and explosiveness. ${ }^{11}$ From T3 to T4, this resulted in a $2.9 \%(\mathrm{Sq})$ and $4.2 \%$ (SQa) drop in the squat. Simultaneously, this resulted in an $8.2 \%$ IPF and $8.8 \%$ IPFa gain. However, DUP did not show this trend from T3 to T4, producing no change in IPF or IPFa, and simultaneously the squat decreased $(\mathrm{Sq}=-7.0 \%, \mathrm{SQa}=-2.6 \%)$. Furthermore, the block method showed a 15\% RFD gain, whereas DUP resulted in a loss $(-22 \%)$ from T3 to T4. Assuming that fatigue negatively affects power development and explosiveness and that these parameters are enhanced during periods of lower fatigue, ${ }^{11,17}$ these data indicate that block was experiencing less accumulated fatigue compared with DUP. Conversely, the DUP showed little alteration in IPF, a loss of squatting strength, and a loss of explosiveness (RFD), suggesting poor fatigue management. These short-term alterations, while not statistically significant, may be quite meaningful. ${ }^{11}$ It is commonly assumed that greater workloads produce superior gains. Equated work is believed to be necessary in order to study the effects of intensity, technique, or other attributes independently ${ }^{12,17}$ and some evidence indicates that higher volumes of work produce greater gains in strength and power. ${ }^{12}$ Although several studies have attempted to equate volume using repetitions,,$^{3,8,9}$ this method is likely the least accurate and reliable method of estimating training volume (work). ${ }^{11,13}$ Furthermore, equated work may obviate innate strengths of the programming model being studied and produce protocols that are not optimum. Assuming VL differences represent work differences, ${ }^{17}$ the present study supports this last point. The block model made statistically equal gains with considerably less work (35\% less VL) and fewer repetitions $(52 \%)$. Thus, total work may not be the most important factor in producing performance gains but rather appropriate training variable manipulation.

A primary purpose of periodization is the removal of linearity. ${ }^{11,18}$ Block protocols ${ }^{19,20}$ are characterized by specific phases that emphasize a particular fitness variable. In this study, the block emphasized strengthendurance (wk 1-3), basic strength development (wk 4-6), and power development (wk 7-10). In contrast, the DUP attempts to accomplish this type of variation using daily alterations; this approach basically entails high volumes of relatively simultaneous training of fitness characteristics, which can be counterproductive. ${ }^{20,21}$

During the first 3 weeks, mean group session time was approximately equal ( $47 \mathrm{~min} / \mathrm{session})$ and this remained constant over the 10 weeks for the DUP; however, during weeks 4-7, the time for the block decreased to about 40 minutes and decreased to about 35 minutes during weeks $8-10$. As training time is a valuable asset, the extra time spent in the weight room for the DUP group also represents a degree of inefficiency. It should be noted that test days occurred on Monday, which was the highest volume day for the DUP, so the actual differences between groups during a typical program could be even greater if the typical training pattern were followed. The increased work undertaken by the DUP group may have resulted in poor fatigue management. ${ }^{10,11,18}$

Previous studies $^{7-9}$ do not appear to manipulate volume and intensity in a manner adequate to produce true heavy and light days of training in either group. A heavy stimulus (ie, high volume/intense training day[s]) followed by adequate recovery time resulting from a light day can produce greater gains than programs using less marked variation. ${ }^{10,11}$ The DUP used RM zones, necessitating frequently training to failure. Most practical periodized training protocols rarely use RMs or RM zones because (1) the use of RM values essentially creates a constant relative maximum effort, reducing variation (ie, no true heavy or light days), and (2) the constant training to failure and the increase in repetitions on light loading days makes fatigue management increasingly difficult, raising the potential for overtraining, ${ }^{22}$ and the potential for strength-power development can be reduced. ${ }^{23}$ Heavy and light days used in the block contributed to the lower $\mathrm{VL}$, and greater performance gain efficiency.

One limitation to this and most previous studies was the short duration (10 wk). Nevertheless, these data indicate that methods of periodized strength power training - structurally similar to the phase potentiation methods described by Stone et al, ${ }^{16}$ the conjugated successive methods of Verkhoshanky, ${ }^{24}$ and the block periodization described by Issurrin ${ }^{19}$ - appear to produce superior effects over a short term. This is particularly true when the efficiency of training is considered.

\section{Practical Applications}

Recently, a number of strength training "periodized models" have been created, with each purported to produce superior results. This study investigated the effects on maximum strength and RFD of 2 methods: block and DUP. The authors of the present study would suggest that block is the superior method, even over a short term (10 wk). This is based on the following.

Training effects are difficult to detect among athletes. ${ }^{16,25}$ For example, differences between first and fourth at the last 6 Olympics, in most sports, have been less than $1.5 \%$. Mujika et al ${ }^{26}$ indicate differences between first and eighth place in the finals of the Olympics can be quite small and are strongly influenced by the type of training program and taper before competition. Thus, small alterations in effect among athletes often have great meaning. Although statistical significance was not achieved, based on ES and percentage changes, the results of this study suggest trends favoring the block similar to those noted by Hartmann et al. ${ }^{4}$ For example, net gains in all forces and RFD favor the block. Conversely, DUP lost performance, which the authors attribute to accumulative fatigue, a similar observation of Apel et al, ${ }^{27}$ after comparing 12 weeks of weekly undulating periodization to a more traditional program. 
Less work was necessary in the block protocol to accomplish statistically similar gains in performance. Furthermore, the additional training time necessitated by DUP protocol (particularly on Monday) can interfere with scheduling in the weight room and practice.

\section{References}

1. Bradley-Popovich GE, Haff GG. Nonlinear versus linear periodization models. Nat Strength Cond Assoc J. 2001;23:42-44.

2. Kraemer WJ, Fleck SJ. Optimizing Strength Training: Designing Nonlinear Periodization Workouts. Champaign, IL: Human Kinetics;2007.

3. Buford TW, Rossi SJ, Smith DB, Warren AJ. A comparison of periodization models during nine weeks with equated volume and intensity for strength. J Strength Cond Res. 2007;21:1245-1250.

4. Hartmann H, Andreas B, Wirth, K. Schmidtbelicher D. Effects of different periodization models on rate of force development and power ability of the upper extremity. J Strength Cond Res. 2009;23:1921-1932. PubMed doi:10.1519/JSC.0b013e3181b73c69

5. Hoffman JR, Ratamess NA, Klatt M, et al. Comparison between different off-season resistance training programs in Division III American college football players. $J$ Strength Cond Res. 2009;23:11-19.

6. Kok L-Y, Hamer PW, Biship DJ. Enhancing muscular qualities in untrained women: linear versus undulating periodization. Med Sci Sports Exerc. 2009;41:1797-1807. PubMed doi:10.1249/MSS.0b013e3181a154f3

7. Peterson MD, Dodd DJ, Alvar BA, Rhea MR, Farve M. Undulation training for development of hierarchical fitness and improved firefighter job performance. J Strength Cond Res. 2008;22:1683-1695.

8. Rhea MR, Ball SD, Phillips WT, Burkett LN. A comparison of linear and daily undulating periodized programs with equated volume and intensity for strength. J Strength Cond Res. 2002;16:250-255.

9. Rhea MR, Phillips WT, Burkett LN, et al. A comparison of linear and daily undulating periodized programs with equated volume and intensity for local muscular endurance. J Strength Cond Res. 2003;17:82-87.

10. Foster C. Monitoring training in athletes with reference to overtraining syndrome. Med Sci Sports Exerc. 1998;30:1164-1168. PubMed doi:10.1097/00005768199807000-00023

11. Stone MH, Stone ME, Sands WA. Principles and practice of resistance training. Champaign, IL: Human Kinetics; 2007.

12. Cronin J, Crewther B. Training volume and strength and power development. J Sci Med Sport. 2004;7:144-155. PubMed doi:10.1016/S1440-2440(04)80004-5
13. Haff GG. Quantifying workloads in resistance training: a brief review. Strength and Cond. 2010;10:31-40.

14. Kraska J, Ramsey MW, Haff GG, et al. Relationship between strength characteristics and un-weighted and weighted vertical jump height. Int J Sports Physiol Perform. 2009;4:461-473. PubMed

15. Nuzzo JL, McBride JM, Cormie P, McCaulley GO. Relationship between countermovement jump performance and multijoint isometric and dynamic tests of strength. $J$ Strength Cond Res. 2008;22:699-707.

16. Stone MH, Sanborn K, O'Bryant HS, et al. Maximum strength-power-performance relationships in collegiate throwers. J Strength Cond Res. 2003;17:739-745.

17. McBride JM, McCaulley GO, Cormie P, Nuzzo JL, Cavill MJ, Triplett NT. Comparison of methods to quantify volume during resistance exercise. J Strength Cond Res. 2009;23:106-110.

18. Bompa T, Haff GG, Tudor O. Periodization of Strength. Champaign, IL: Human Kinetics; 2009.

19. Issurin, V. Block periodization versus traditional training theory: a review. J Sports Med Phys Fitness. 2008;48:6575.

20. Garcia-Pallares J, Garcia-Fernandez M, Sanchez-Medina $\mathrm{L}$, et al. Performance changes in world-class kayakers following two different training periodization models. Eur J Appl Physiol. 2010;110:99-107. PubMed doi:10.1007/ s00421-010-1484-9

21. Breil FA, Simone N, Weber-SK, et al. Block training periodization in alpine skiing: effects of 1-day HIT on $\mathrm{VO}_{2 \max }$ and performance. Eur J Appl Physiol. 2010;109:10771086. PubMed doi:10.1007/s00421-010-1455-1

22. Campos GE, Luecke TJ, Wendeln HK, et al. Muscular adaptations in response to three different resistancetraining regimens: specificity of repetition maximum training zones. Eur J Appl Physiol. 2002;88:50-60.

23. Izquierdo M, Ibanez J, Gonzalez-Badillo JJ, et al. Differential effects of strength training leading to failure versus not to failure on hormonal responses, strength, and muscle power gains. J Appl Physiol. 2006;100:1647-1656. PubMed doi:10.1152/japplphysiol.01400.2005

24. Verkhoshansky YV. Fundamentals of Special Strength Training in Sport. Moscow: Fizkultura i Sport; 1977.

25. Hopkins WG, Marshall SW, Batterham AM, Hanin J. Progressive statistics for studies in sports medicine and exercise science. Med Sci Sports Exerc. 2009;41:3-13. PubMed

26. Mujika I, Padilla S, Pyne D. Swimming performance changes during the final 3 weeks of training leading to the Sydney 2000 Olympic Games. Int J Sports Med. 2002;23:582-587.

27. Apel JM, Lacey RM, Kell RT. A comparison of traditional and weekly undulating periodization strength training programs with total volume and intensity equated. J Strength Cond Res. 2011;25:694-703. PubMed 
Copyright of International Journal of Sports Physiology \& Performance is the property of Human Kinetics Publishers, Inc. and its content may not be copied or emailed to multiple sites or posted to a listserv without the copyright holder's express written permission. However, users may print, download, or email articles for individual use. 